\title{
Assessment of Supervised Drug Release in Cordial Embedded Therapeutics
}

\author{
By Adel Razek ${ }^{*}$
}

This paper targets to illustrate an assessment strategy of implanted therapeutics for restricted drug delivery with minimally invasive and non-ionizing user-friendly conditions. A functioning analysis indicates an image guided solution involving magnetic resonance imager (MRI). The paper then discusses MRI and investigates its environmental compatibility for embedded therapeutics. This investigation accounts for the nature of the different electromagnetic fields of the imager. We have also investigated the confrontation of the imager performance, the patient wellbeing and biological effects. We have verified the MRI environmental compatibility through EMC analysis of field perturbation due to implanted materials considering the conventional imager operation. EMC analysis $3 D$ numerical applications support the contribution at the end of the paper. This study is a mixed of survey and modeling.

Keywords: Implants, Minimally Invasive, Non-Ionizing, Imaging, EMC Analysis

\section{Introduction}

A recent significant challenge concerns embedded therapeutics (Dash et al. 1998, Perry et al 2007, Bazaka et al. 2013, Amar et al. 2015, Eltorai et al. 2016, Ramachandran et al. 2017, Mao et al 2018).

Beside, some are using active locally restricted drug release technologies (Massiota et al. 2018, Okabe et al. 2003, Hsu et al. 2016, Hsu et al. 2014).

Embedded therapeutics constructions might use local treatment minimally invasive technologies with implants. These implants utilize drug delivery systems for intra or adjacent tissue covering a given zone.

Friendly wellbeing and security of patient infer minimally invasive technology. For embedded therapeutics, this indicates the use of biodegradable materials and wireless controlled actuation of implants.

Biodegradable structures are used in order to bypass a second surgical elimination of the devices and to avoid a recurring immune reaction of the external body (Okabe et al. 2003, Hsu et al. 2016, Hsu et al. 2014, Yun et al. 2009, Boutry 2012).

Wireless powering and control transfer consists of transferring energy and signals from an external source to the load without physical contact (Boutry 2012, Ding et al. 2014).

For kindly comfort of patient, the therapy should be accurately restricted to the affected zone. The accuracy of the restricted zone of therapy is related to the precision of implant actuation and hence the wireless transmission circuit. This accuracy is also much correlated to the exactitude of the implant tracking in the

*Emeritus Research Director, C.N.R.S. \& Honorary Professor, CentraleSupelec, GeePs, University of Paris-Saclay and Sorbonne University, France. 
space. Therefore, the adequate key for such high-resolution topological survey is the image guided position detection of the implant in the space (Razek 2018).

This paper relates the details and the discussion of the different features covering the wellbeing and the security of patient in image guided embedded therapeutics. After the adoption of an adequate imager, the study will relay the performance and the environmental compatibility of the elements involved in the projected system. These concerns in particular the details of the elected imager constitutes as well as the basis of its functioning. These details, which are necessary for the achievement of the followed assessment strategy, would help in deepening the investigations developments. The paper ends by EMC computation examples supporting the imager environmental compatibility of the elements involved in the projected system.

\section{Implanted Device and Imaging}

As discussed in the introduction, the minimally invasive technology and the accurately restricted drug delivery suggest an image guided drug release embedded implant that one can power and control wirelessly by an external source. Such device may consist of an interactive system operating autonomously if integrates an artificial intelligence AI algorithm as shown in figure 1. Such system is supposed to operate under the survey of the medical team (Razek 2018).

Figure 1. Interactive Autonomous Image Guided Drug Release System

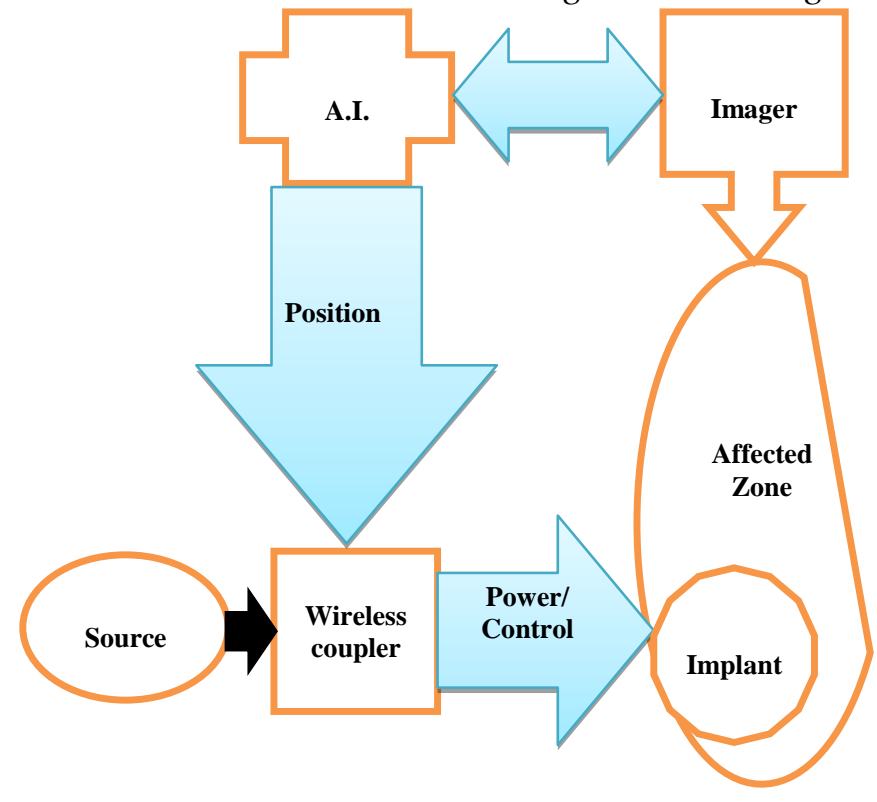

Non-ionizing Imaging

Different biomedical imaging technologies are available; they employ either, X-rays, sound, magnetism (Magnetic Resonance) or radioactive pharmaceuticals. 
Each of these issues is more or less suitable for a specified situation. For conventional imaging circumstances where the exposure to the imager arrangement is limited in time interval there is no constraints relative to the nature of these imagers. Therefore, the problem of the ionizing radiations concerning some of these imagers does not impose any serious constraint.

In case of sustained exposure to the imager as in the case of image assistance therapeutic, only the magnetic resonance imager (MRI) and the ultrasound imager do not suffer of the ionizing radiations serious drawback (Tsekos et al. 2007). However, it is noteworthy that the ultrasound imager although radiation free, due to its functioning principle, it necessities an acoustic window (without air or bones).

The local delivery implant technology ought to use non-destructive biological and health undistinguished sensing. Thus, MR image guided technology seems to be an excellent and universal sensing candidate for embedded therapeutics (Razek 2018).

On the other hand, the use of the MR imager in image guided therapeutics as well as in other medical interventions assisted by imaging, implies two types of restrictions concerning its performance and environmental compatibility. This is due to the practice context of the imager concerning the treatment time interval and the proximity of the tools, necessary for the treatments, to the imager environment.

Concerning the treatment time interval, one notes that the time necessary to obtain an image is directly in relation to the performance of the imager and in particular, to the output of its gradient coils; we will discuss this point later in the paper. Higher the gradient output, the time necessary for an image is shorter, which is better for the outcome of the treatment. In the meantime, such high output increases the risk of negative biological effects for the patient. Therefore, we face a problem of finding the middle ground between the imager performance and the harmful biological effects.

For the proximity of treatment tools, it is worth mentioning that MR imager is very sensitive in connect with the electromagnetic noise and the introduction of certain external stuffs as magnetic or conductor materials in the imager environment; we will discuss this compatibility aspect in the next section.

\section{Treatments Tools and Constraints in MR Imagers}

One of most commonly used diagnostic tools is the MRI technology. Its use in a context gathered in therapeutic image assisted set, permits to achieve an excellent localising tool improving the therapeutics and allowing precise minimally invasive procedures under non-ionising environment. Thus, accomplishing a significant impact on clinical determinations and health consequences. Additional social challenges, in complement to the efficacy of image guided interventions and therapeutics, in different up-and-coming arenas of biomedical research are outstanding. For example, mapping of brain activity in neuroscience investigations and supervising advancement of reintegration therapies.

The Magnetic resonance imaging (MRI) technique delivers images of the 
biological tissue conditional on electromagnetic field. These images acquired throughout the arrangement of three kinds of electromagnetic fields: an intense static magnetic field, a low frequency pulsed little static magnetic space varying field (gradient), and a high radio frequency field. In a condensed manner, the tissue under an arrangement of field acquaintance, returns information that converted into signals that once again translated into images.

The MRI technique operates, as mentioned before, different electromagnetic fields with very different magnitudes and frequencies. The arrangements using such technology are very susceptible to electromagnetic noise and to the existence of magnetic or conductive substances that can produce image degeneration. Subsequently, actuating and mechatronic systems usually used in image guided interventions and therapeutics are not appropriate for MRI appliances, giving rise to a need for adequate type of schemes: MR compatible. Some MRI compatible specialised robots and micro-robots designed for particular interventions and therapeutics exist (N.V. Tsekos, et al 2007). We have to use magnetic released materials in the construction of robots that use distinct forms of actuation, which are MRI compatible.

The in depth analysis of the environmental compatibility as well as the confrontation of the performance and the constraints in MR image guided technology concerns the next sections. Due to this aim, one needs understanding the basic principle of such imager as well as going inside details of its components.

\section{MRI Basis, Fields and Components}

Due to clinical circumstances, MRI is principally utilized in favor of imaging of hydrogen (or further specifically the hydrogen nucleus that is a proton) confined in human body. Hydrogen stand in for $63 \%$ of the atoms of the whole body. A proton is a mass with a positive charge, which turns around an axis on itself. One can characterize its magnetic moment under the arrangement of a revolving vector on itself: this typifies the spin of the proton.

It is nevertheless likely to accomplish MRI using further nuclei than hydrogen, for instance phosphorus or sodium; however, their unimportant intensity in living tissues marks their revealing problematic in exercise.

Without a doubt, in human body, protons have arbitrarily orientations. Therefore, their resultant magnetic field is zero, and the living body does not present any magnetization. On the other hand, they do not revolve together; in other words, they behave out of phase.

Considering the MRI principle, the protons could do with three necessary arrangements in the interior of the examined part of the living tissue (S): bring into line all the protons in an unchanged direction, make them take tums simultaneously together in the volume $\mathrm{S}$, and exactly so locate their distinct spatial origin.

\section{Fields Utilized in MRI}

As mentioned before MRI functioning principle imposes three necessary 
conditions.

The first arrangement consisting of aligning all the protons in the volume $S$ could be achieved by placing the part $S$ of the patient in a strong magnet to guide all protons in the axis of the static magnetic field $\mathrm{B}_{0}$ of this magnet.

Considering the second one, to make the protons of the volume $\mathrm{S}$ rotate together we can supply them by an energy (excitation) under the form of a radio frequency wave $\mathrm{B}_{1}$; the frequency of such wave should be identical to the natural frequency of rotation of protons (resonance). The value of this natural frequency $\mathrm{f}_{\mathrm{L}}$ (Larmor frequency of protons) which is dependent of $\mathrm{B}_{0}$, equals $42.5 \mathrm{MHz}$ per tesla. The provided energy to the protons of $\mathrm{S}$, not only synchronizes their rotation due to resonance phenomenon, but as well, fashions the ways of protons rocking a bit related to the axis of the magnet. The radio frequency wave disruption (relaxation) outcomes in the break out of axis rocking and synchronous revolution of the protons of the volume $\mathrm{S}$. Associated to this phenomenon one can characterize the operation by two relaxation times respectively $T_{1}$ and $T_{2}$. Thus, go again to the original state, so the protons inside the volume $S$ bring back energy that provided to them by the radio frequency wave. This restored energy is still in the form of a wave. It is likely to sense such a signal through an appropriate tuned radio frequency coil (antenna).

In the third arrangement, if one accurately locates the spatial origin of the signal, it is thinkable from there to institute a matrix image; a physical 3D location mapping of the volume $S$ protons. Regarding such spatial localization, for each of the sections of spin in the volume $\mathrm{S}$, it is possible to go through a distinctive magnetic field value. Thus, we would be capable to image their positions. To accomplish this we apply a 3D space gradient function under the form: $G(x, y, z)$ on the static magnetic field $\mathrm{B}_{0}$ in the volume $\mathrm{S}$. Therefore, engendering a continuing space variant in $\mathrm{B}_{0}$ will outcome in a space magnetic field $\mathrm{B}_{0}(\mathrm{x}, \mathrm{y}, \mathrm{z})=$ $\mathrm{B}_{0}+\mathrm{G}(\mathrm{x}, \mathrm{y}, \mathrm{z})$, matching a space Larmor frequency of protons $\mathrm{f}_{\mathrm{L}}(\mathrm{x}, \mathrm{y}, \mathrm{z})$, allowing coding spatial image in the volume $\mathrm{S}$. The obtained gradients are under the form of pulsations with a very low frequency repetition that produce a kind of shouting "Hammering". The radio frequency coil (antenna) being tuned to the space Larmor frequency of protons $f_{L}(x, y, z)$ in the volume $S$ and matched (impedance adapted, classically to $50 \mathrm{Ohms}$ ). This frequency is variable in the volume $\mathrm{S}$ and generally one tune the coil to the frequency in compliance to the field applied in the center of the volume $S$.

From the above lines, we get the message that the MR imaging system use the services of three different electromagnetic fields to get hold of images of the inspected fragment of the body S. It is noteworthy that the natures of these three fields are completely different concerning the amplitude and the frequency as well as the presence during functioning. Under the classical operation of a MRI to insure the correct functioning of the imager, we have to protect and compensate both the static and the gradient fields. The radiofrequency field is the most vulnerable notably against electromagnetic perturbations and the introduction of certain external matters in the imager environment. 


\section{MRI Components}

As mentioned in the last section, an MR imager has three indispensable components producing the stated three fields: magnet, gradient and radiofrequency. The majority of magnets are of the superconducting type whose responsibility is to engender a homogeneous static magnetic field inside the bore $\mathrm{B}_{0}$. Three couples of gradients coils are acting in the three axes $\mathrm{x}, \mathrm{y}$, and $\mathrm{z}$ directions that make available the encoding of the space inside the bore. The radiofrequency coils produce electromagnetic fields at the Larmor frequency $\mathrm{B}_{1}$.

The fields established by MRI components have different performs and belong in distinctive scales in terminologies of strength, frequency and duration. Because of these separate extents, it is conceivable to study on, a case-by-case basis, each of the three fields. .

It is possible to classify the radiofrequency coils or antennas into two sorts: surface and volume ones. The radiofrequency field $\mathrm{B}_{1}$ is by principle in perpendicular orientation with that of the static field $\mathrm{B}_{0}$. Comparing the two types of antennas, the volume one (called often birdcage) has a most important advantage that produces a uniform field inside the coil. As stated formerly an MRI is very susceptible to electromagnetic noise that can instigate image worsening. The insertion of magnetic or conductive stuffs in the neighboring environment of MRI could bring in such perturbation.

In the meantime, the electromagnetic environment that reflected unsympathetic, with intense static field, to which is place over high frequency and pulsated fields. We can define the MRI compatibility in relation to such environment. The device introduced in the MRI hosting must not disturb the field while it is in place, with the electronics powered and it is running. This demonstrates that we have to choose the device constituting materials judiciously, the electronics used must be of low pollution and the whole system optimized. Yet, investigational studies have shown that the insertion of small ferromagnetic or conductive parts (nuts, screws ...) could almost not disturb imaging. This is due to the conventional protection and compensations of the static and gradient fields as mentioned before. Finally, despite these constraints, the realized device must have a sub-millimeter resolution, which necessitates the employment of an adequately rigid stuff and appropriately precise actuation.

Take into account these requirements; we can design MRI compatible specialized robots constructions for particular interventions or therapeutics. These robots have to use MRI compatible actuation. Outstanding actuation applicants, particularly in medical therapeutics and chirurgical interventions, are using active smart materials (e.g. piezoelectric and magnetostrictive) actuators due to their supremacy in terms of accuracy, rapidity and mass.

Enhancement in the domain of image guided therapeutics and interventions count on the expansion, elucidation and acceptance of accessible MRI, actuators and robots for medical therapeutics and chirurgical make easier. Each of these constituents performs for a complicated equipment. The bringing together of these components to establish a new diagnostic tool supporting therapeutics and interventions ask for supplementary convoluted technologies. The investigation 
and analysis of such gathered device will allow defining imperatives covering the compatibility of the diverse constituents despite the fact that keep in good state the efficiency and dismissing the added cost of each one. Therefore, one can accomplish a negotiation of could do with compatibility extent respects within acceptable limits efficiency and extra cost. Because of these regards, one can enhance the clinical outcomes founded on the suggested diagnostic setting allowing milder health consequences for the patients and the medical staff, while be part of the cause of the sustainability of the health care organization.

\section{MR Image Guided Therapeutics}

The use of MRI in a context gathered in therapeutic assisted set, as stated before, permits to accomplish an excellent localizing tool refining image-based therapeutics and permitting accurate minimally invasive processes under nonionizing environment. Thus, undertaking a substantial impact on clinical determinations and health consequences. On the other hand, the MRI even though radiation free, its operation must carry out high performance and in the same time low biological effects. In addition, the tools necessary for treatment should comply with the imager environmental compatibility.

\section{MRI Performance and Biological Effects}

Each of the three fields engaged in the MRI scheme link up with the physical parameters of biological matters.

Conceivably the securest one of the imager magnetic fields is the static one. From the existing literature, only the existence of ferromagnetic stuffs or pacemakers in the body of patients could engender wellbeing risks notably related to the contact with $\mathrm{B}_{0}$

The second likely provider of risk of imager fields is the pulsated field gradients. Due to the augmenting request for ephemeral cycles with larger temporal and spatial resolutions, the renovation of up to date gradient structures has led in the latest years to amplify considerably the structure output (strength and slew rate) to reduce imaging time. Regardless of this, it is commanding to set appropriate edges for this gradient output to comprehend the arrival of disagreeable side effects for the patient. High output gradients provoke a oscillating electric field that may produce unlikable peripheral nerve stimulation (PNS) and/or cardiac stimulation. These electric fields, if of enough strength and duration, can instigate the excitation of the peripheral nervous system with symptomatically spatially restricted feelings of compression, irritating, or muscle astringent. On the other hand, PNS, which can be embarrassing even if not alarming to the patient, has a limit poorer than the strength expected for possibly life-menacing cardiac stimulation (Razek 2018). It is noteworthy that from the above lines we are in presence of an optimization challenge. The high imager performance related to its high output gradient coil is supposed to lead to shorter imaging time. This impulse in the direction of the wellbeing of the patient. In the meantime, this high output 
could cause harmful biological effects which thrusts in the opposite direction of the patient wellbeing.

Radio frequency power amassing matches to the greatest danger for patient security in MR imager exams. The specific absorption rate (SAR) functioned in MRI inspections could be of $9 \mathrm{~W} / \mathrm{kg}$ and for short sufficient interval so as generating less than $1{ }^{\circ} \mathrm{C}$ inside body temperature rise. A local growth in temperature of $1{ }^{\circ} \mathrm{C}$ in a healthy subject is totally out of hazard; thus, the MRI SAR ranks are below the limits announced by international security references.

This debate displays that awareness of the security of magnetic resonance imaging schemes can aid optimize both the safety and effectiveness of structure process. Characteristic values typifying the three different fields in MRI (Intensity, frequency and duration) (Tsekos et al. 2007) are:

$\mathrm{B}_{0}: 0.2-7 \mathrm{~T}, 0 \mathrm{~Hz}$, Always present

Gradient: 0-50 mT/m, 0-10 kHz, multiple pulses of few ms

$\mathrm{B}_{1}$ : 0-50 $\mu \mathrm{T}, 8-300 \mathrm{MHz}$, Amp. Mod. Pulses of few ms

\section{MRI and Environmental Compatibility}

Magnetic resonance imaging (MRI) system employs static magnetic field, magnetic field gradients and fast evolution of radio frequency pulses.

The static magnetic field should be homogeneous and the magnetic field gradient (location dependent magnetic field) should be controlled and uniform. These two fields need compensation and correction to meet these conditions for conventional operation of the imager. The radio frequency wave has a frequency identical to this of rotation of protons (Larmor frequency of protons) which is dependent on the static field value and given by $42.5 \mathrm{MHz}$ per tesla.

The MRI device is very sensitive to electromagnetic noise that can cause image deterioration. The introduction of magnetic or conductive materials in the close environment of MRI contributes significantly to such electromagnetic noise. The MRI-compatibility definition is in relation to such environment.

Therefore, in embedded therapeutics the implant is supposed to employ MRIcompatible materials and must utilize MRI-compatible actuation (Razek 2018, Tsekos et al. 2007, Chinzei et al. 1999, Khairi et al. 2016, Hariri et al. 2014, Su et al. 2018, Hariri et al. 2015, Khan et al. 2016, Dagdeviren et al. 2016, Lemair et al. 2015, Stapleton et al. 2017).

\section{EMC Analysis and Formulation}

Electromagnetic compatibility - EMC analysis aims to determine the impact of introducing in the MRI environment different materials and structures used in embedded therapeutics. For EMC analysis, we could consider different electromagnetic mathematical formulations and techniques (Ren et al. 2000, Ouchetto et al. 2007, Ren et al. 1990).

Expressions (1-4) give the basic full-wave electromagnetic formulation 
(Razek 2018):

$$
\begin{aligned}
& \nabla \times \mathbf{H}=\mathbf{J} \\
& \mathbf{J}=\sigma \mathbf{E}+j \omega \mathbf{D}+\mathbf{J}_{\mathrm{e}} \\
& \mathbf{E}=-\nabla \mathbf{V}-j \omega \mathbf{A} \\
& \mathbf{B}=\nabla \times \mathbf{A}
\end{aligned}
$$

Where $\mathbf{H}$ and $\mathbf{E}$ are the magnetic and electric fields, $\mathbf{B}$ and $\mathbf{D}$ are the magnetic and electric inductions, $\mathbf{A}$ and $\mathrm{V}$ are the magnetic vector and electric scalar potentials. $\mathbf{J}$ and $\mathbf{J}_{\mathrm{e}}$ are the total and source current densities, $\sigma$ is the electric conductivity and $\omega$ is the frequency pulsation.

The 3-D solution of ( 1 to 4$)$ permits to determine the perturbations in electromagnetic fields due to the introduction of external matters in a given system for a frequency pulsation (Wang 2017). Such impact permits to check the bounds of the system operation alteration (Razek 2018).

\section{Application in MRI Environment}

The analysis described in the last section could be achieved with the help of discretized 3-D finite element model (Ding et al. 2014, Khairi et al. 2016). Considering compensated static field and gradient field as described before, the interaction of different hosted materials constituting the embedded implant in the MRI environment with radio frequency magnetic field has been investigated (Razek 2018).

\section{Simulation Conditions}

The simulation system consists of a birdcage coil of $30 \mathrm{~cm}$ diameter and 30 $\mathrm{cm}$ length embedding a material or a structure of a cubic form of $5 \times 5 \times 5 \mathrm{~cm}^{3}$, placed inside a $60 \mathrm{~cm}$ diameter tunnel. The birdcage coil generates a radio frequency field (at frequency $63.87 \mathrm{MHz}$ ). This frequency corresponds to the Larmor frequency of protons in a static field of $1.5 \mathrm{~T}$.

One can compute the radio frequency field distribution in the tunnel using 3D Edge Finite Elements discretization of the field E. To take into account the skin effect in case of conductor, a surface impedance boundary condition is used. The system's boundary (tunnel bore) is considered as a perfect conductor in which the field cannot penetrate.

We can check the different materials composing embedded implants including their actuation with the help of the simulation system. 
Numerical Results

In a previous work (Khairi et al 2016) concerning robotized interventions, we have verified the use of piezoelectric actuators in robot arms for multilayer actuators composed of PZT layers and aluminum plates with a very trivial thickness. The results confirmed that one could significantly reduce the perturbation, mainly due to conductors, by adjusting the position of the actuator axis relative to the field direction.

Concerning the present work we consider two categories of materials which are commonly used in implant actuation: piezoelectric and conductor materials of organic or non-organic nature. We can calculate the magnetic field distribution inside the tunnel for two examples of these materials. The first is a piezoelectric $\left(\mu_{\mathrm{r}}=1.0, \varepsilon_{\mathrm{r}}=[450990990], \sigma=0 \mathrm{~S} / \mathrm{m}\right)$. We note that the piezoelectric permittivity is a vector (anisotropic) whose value is smaller in polarization direction than the two other directions. The second is a conductor $\left(\mu_{\mathrm{r}}=1.0, \varepsilon_{\mathrm{r}}=1.0, \sigma=\right.$ 3.77e7 S/m).

Figure 2 shows the radiofrequency magnetic field (vertically directed) distribution in the axial cross section of the birdcage inside the tunnel for the reference case: no material.

Figure 2. Radiofrequency Magnetic Field (Vertically Directed) Distribution in the Reference Case: no Material

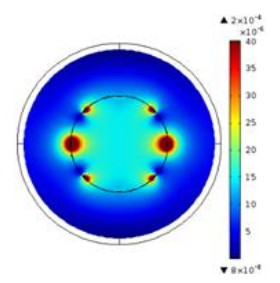

Figure 3 shows the magnetic field distribution for the case of introducing a $5 \times 5 \times 5 \mathrm{~cm}^{3}$ piezoelectric material volume. It shows, compared to the reference case, that the piezoelectric has a small effect on the field (very small values of displacement currents).

Figure 3. Radiofrequency Magnetic Field (Vertically Directed) Distribution in the Case of Piezoelectric Material

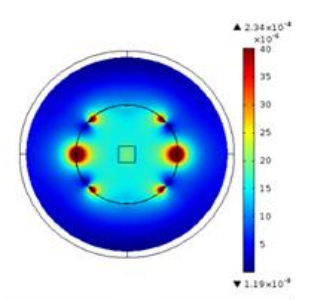

Figure 4 shows the magnetic field distribution for the case of introducing a conductor material volume. It shows, compared to the reference case that the conductor modifies significantly the field distribution due to the induced eddy currents in the conductor. 
Figure 4. Radiofrequency Magnetic Field (Vertically Directed) Distribution in the Case of Conductor Material

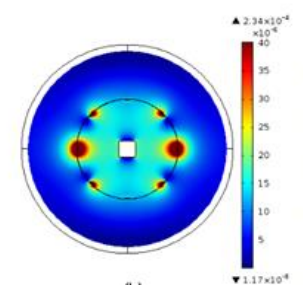

It is noteworthy that the degree of perturbation of the field in the conductor case is proportional to the significance of the conductor surface perpendicular to the field direction.

Investigating the relation between the degree of perturbation of the field due to the conductor and the surface of this last perpendicular to the field direction, the case of a non-conductor material coated by very small thickness plates of conductors, has been considered. In practice, this is the case of electrodes (conductor) to allow the supply of energy (Hariri et al. 2014, Su et al. 2018, Hariri et al. 2015).

We considered the case of a piezoelectric material as in figure 3 but coated on 2 opposite faces by conductor thin electrodes. We have computed the field distributions for the two cases where the electrodes are perpendicular and parallel to the field direction. Figure 5 shows the field distributions for these cases. The results confirmed that the direction of the conductor plays an important role. We can reduce significantly the impact of the conductor when it is parallel to the field.

Figure 5. Radiofrequency Magnetic Field distribution in the Case of Piezoelectric with Electrodes (a) Material Configuration (b) Field Distribution when Conductors are Perpendicular to the Field (c) Field Distribution when Conductors are Parallel to the Field

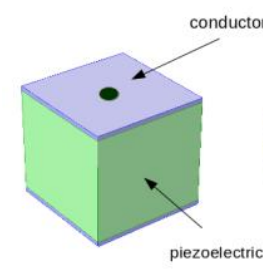

(a)

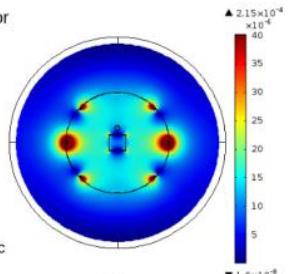

(b)

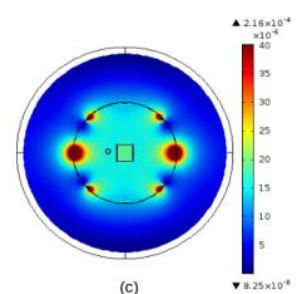

\section{Conclusion}

In this work that is a mixed of survey and modeling, we have investigated the issues of minimally invasive technology, accurately restricted drug delivery and non-ionizing detection in implanted therapeutics. The result suggests an image guided interactive solution with MR imager. The MRI compatibility of the embedded materials is then considered. We have also discussed the compromise of the imager performance, patient wellbeing and harmful biological effects. 
For the sake of MRI compatibility, we have performed an EMC analysis considering the nature of different MRI fields and their conventional protections and corrections. We have investigated the impact of the presence of different matters composing implants in MRI environment. The first results elucidate that organic or non-organic piezoelectric and conducting materials could be tolerable in MRI environment by handling their space locating in the field orientation.

Future research focuses on the nature and size of materials involved in implanted therapies that recognize the well-being and safety of the patient. At the same time, these materials have a duty regarding acceptance in the MRI environment.

Concerning the nature of these materials, it can be generally of the active type (smart materials). The external source of wireless excitation acting on these materials can be a field (magnetic or electric) of low intensity so as not to disturb the operating fields of the imager. An open question of research concerning such a field of excitation, could it be linked to the operating fields of the imager? This is a delicate case with regard to the sequences and scales of these fields as well as their possible disturbances.

\section{References}

Amar AB, Kouki AB, Cao H (2015) Power approaches for implantable medical devices. Sensors 15(11): 28889-28914.

Bazaka K, Jacob MV (2013) Implantable devices: issues and challenges. Electronics 2(1): $1-34$.

Boutry C (2012) Biodegradable passive resonant circuits for wireless implant applications. Dissertation for the degree of Doctor of Sciences, Submitted to ETH Zurich.

Chinzei K, Kikinis R, Jolesz FA (1999) MR compatibility of mechatronic devices: design criteria. MICCAI (1679): 1020-1030.

Dagdeviren C, Joe P, Tuzman O L, Park KI, Lee KJ, Shi Y, Huang Y, Rogers JA (2016) Recent progress in flexible and stretchable piezoelectric devices for mechanical energy harvesting, sensing and actuation. Extreme Mechanics Letters 9(1): 269-281.

Dash AK, Cudworth GC (1998) Therapeutic applications of implantable drug delivery systems. Journal of Pharmacological and Toxicological Methods 40(1): 1-12.

Ding PP, Bernard L, Pichon L, Razek A (2014) Evaluation of electromagnetic fields in human body exposed to wireless inductive charging system. IEEE Transactions on Magnetics 50(2): 1037-1040.

Eltorai AE, Fox H, McGurrin E, Guang S (2016) Microchips in medicine: current and future applications. BioMed Research International Article ID 1743472: 7.

Hariri H, Bernard Y Razek A (2014) A traveling wave piezoelectric beam robot. Smart Materials and Structures 23:2.

Hariri H, Bernard Y, Razek A (2015) Dual piezoelectric beam robot: the effect of piezoelectric patches' positions. Journal of Intelligent Material Systems and Structures 26(18): 2577-2590.

Hsu YH, Chen DW, Tai CD, Chou YC, Liu SJ, Ueng SW, Chan EC (2014) Biodegradable drug-eluting nanofiber-enveloped implants for sustained release of high bactericidal concentrations of vancomycin and ceftazidime: in vitro and in vivo studies. International Journal of Nanomedicine 9(1): 4347-4355. 
Hsu YH, Lin C, Yu Y, Chou Y, Liu S, Chan E (2016) Dual delivery of active antibactericidal agents and bone morphogenetic protein at sustainable high concentrations using biodegradable sheath-core-structured drug-eluting nanofibers. International Journal of Nanomedicine 2016(11): 3927-3937.

Julien Massiota et al (2018) Lipid-porphyrin conjugates as potential multifunctional drug delivery system. International Symposium on Drug Discovery and new Therapeutics France, April 9-11.

Khairi R, Razek A, Laurent B, Corcolle R, Bernard Y, Pichon L, Poirier-Quinot M, JeanChristophe G (2016) EMC analysis of MRI environment in view of Optimized performance and cost of image guided interventions. International Journal of Applied Electromagnetics and Mechanics 51(1): S67-S74.

Khan A, Abas Z, Kim HS, Kim J (2016) Recent progress on cellulose-based electro-active paper, its hybrid nanocomposites and applications. Sensors 16(8).

Lemaire E, Moser R, Borsa CJ, Shea H, Briand D (2015) Green paper-based piezoelectric material for sensors and actuators. Procedia Engineering 120: 360- 363

Mao S, Wang H, Mao ZH, Sun M (2018) A miniature implantable coil that can be wrapped around a tubular organ within the human body. AIP Advances 8(5).

Okabe J, Kimura H, Kunou N, Okabe K, Kato A, Ogura Y (2003) Biodegradable intrascleral implant for sustained intraocular delivery of betamethasone phosphate. Investigative Ophthalmology \& Visual Science 44(2): 740-744.

Ouchetto O, Zouhdi S, Bossavit A, Griso G, Miara B, Razek A (2007) Homogenization of structured electromagnetic materials and metamaterials. Journal of Materials Processing Technology 181 (1-3): 225-229.

Perry J, Chambers A, Spithoff K, Laperriere N (2007) Gliadel wafers in the treatment of malignant glioma: a systematic review. Journal of Current Oncology 14(5): 189-194.

Ramachandran R, Junnuthula VR, Gowd GS, Ashokan A, Thomas J, Peethambaran R, Thomas A, Unni AK, Panikar D, Nair SV, Koyakutty M (2017) Theranostic 3dimensional nano brain-implant for prolonged and localized treatment of recurrent glioma. Scientific Reports 7:43271.

Razek A (2018) Towards an image guided restricted drug release in friendly implanted therapeutics. The European Physical Journal Applied Physics 82(3).

Ren Z, Razek A (1990) New technique for solving three-dimensional multiply connected eddy-current problems. IEE Proceedings A - Physical Science, Measurement and Instrumentation, Management and Education 137(3): 135-140.

Ren Z, Razek A (2000) Comparison of some 3D eddy current formulations in dual systems. IEEE Transactions on Magnetics 36 (4): 751-755.

Stapleton A, Noor MR, Sweeney J, Casey V, Kholkin AL, Silien C, Gandhi AA, Soulimane T, Tofail SAM (2017) The direct piezoelectric effect in the globular protein lysozyme, Applied Physics Letters 111 (14).

Su Q, Quan Q, Deng J, Yu H (2018) A quadruped micro-robot based on piezoelectric driving. Sensors 18(3).

Tsekos NV, Khanicheh A, Christoforou E, Mavroidis C (2007) Magnetic resonancecompatible robotic and mechatronics systems for image guided interventions and rehabilitation: A Review Study. Annu. Rev. Biomed. Eng. 9(1): 351-387.

Wang ZL, (2017) On maxwell's displacement current for energy and sensors: the origin of nanogenerators. Materials today 20(2): 74-82.

Yun Y, Dong Z, Lee N, Liu Y, Xue D, Guo X, Kuhlmann J, Doepke A, Halsall HB, Heineman W, Sundaramurthy S, Schulz MJ, Yin Z, Shanov V, Hurd D, Nagy P, Li W, Fox C (2009) Revolutionizing biodegradable metals. Materials today 12(10): 2232. 
